\title{
Archéopages
}

Archéopages

Archéologie et société

\section{Étables à bovins, écuries, bergeries, porcheries. Manuels agronomiques et vestiges médiévaux et modernes en Île-de-France}

Jean-Yves Dufour

\section{(2) OpenEdition \\ Journals}

Édition électronique

URL : https://journals.openedition.org/archeopages/305

DOI : 10.4000/archeopages.305

ISSN : 2269-9872

Éditeur

INRAP - Institut national de recherches archéologiques préventives

Édition imprimée

Date de publication : 1 mai 2013

Pagination : 60-66

ISSN : 1622-8545

\section{Référence électronique}

Jean-Yves Dufour, «Étables à bovins, écuries, bergeries, porcheries. Manuels agronomiques et vestiges médiévaux et modernes en Île-de-France », Archéopages [En ligne], 35 | 10/2012, mis en ligne le 01 octobre 2014, consulté le 21 janvier 2022. URL : http://journals.openedition.org/archeopages/ 305 ; DOI : https://doi.org/10.4000/archeopages.305 


\section{Étables à bovins, écuries,}

bergeries, porcheries

Manuels agronomiques et vestiges

médiévaux et modernes en Île-de-France

Jean-Yves Dufour Irrap, UMR 7041 « Archeologie et Sciences de lantiquité,

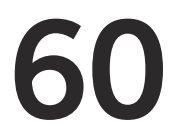

1. Selon l'expression de Claude Lévi-Strauss.
L'observation des fermes franciliennes traditionnelles nous place devant une évidence : 80 à $90 \%$ du bâti d'une exploitation est destiné à abriter et à gérer les récoltes, l'équipement, l'outillage (machines, attelages) et à loger le bétail. Les usages ont varié d'une période culturelle à l'autre, et d'une région à l'autre. On peut néanmoins envisager qu'une grande partie des vestiges bâtis étudiés lors des fouilles en contexte rural ou villageois, correspondent non pas à des habitations, mais bel et bien à des locaux destinés à la resserre et au logement animal. Par la nature des aménagements et des rejets qui leur sont associés (foyer, silo, four, latrines...), les archéologues reconnaissent aisément les habitations humaines. Il est moins aisé d'identifier le logement animal, surtout quand celui-ci est fortement «humanisé», comme c'est le cas du chien. La difficulté paraît telle qu'elle peut conduire à nier l'utilité et donc l'existence de logements pour les principaux bétails, au profit de la rusticité et de l'adaptation bien connue de certaines races animales aux frimas hivernaux... (Rozier, 1785, p. 71 ; Digois, 1942, p. 103). Sur la majorité du territoire français, les observations ethnographiques, archéologiques et archivistiques témoignent sans équivoque de la nécessité d'abriter les animaux pour mieux les protéger en certaines périodes (hiver, mise bas, maladie), et mieux les exploiter (multiplication, engraissement...). «Entre des étuves vicieuses et le plein air toute l'année, il faut trouver le meilleur logement», conclut Perthuis de Laillevault dans son Traité d'architecture rurale (1810). Nous ne traiterons dans cet article que du logement des principaux bétails d'une ferme, à savoir les bovins, les chevaux, les caprinés (le mouton et la chèvre) et le porc. Le logement du bétail a-t-il une réalité archéologique, ou bien peut-il être confondu, mêlant chevaux et moutons sous le même toit? L'enjeu est de distinguer en fouille archéologique une bergerie, une porcherie, une écurie et une étable à bovin.

\section{Questionnements agronomiques appliqués à l'archéologie}

Si le site le permet, les géoarchéologues mettent parfois en œuvre des analyses de phosphore pour repérer des zones de stabulation ou d'épandage des fumiers (voir l'article de Broes et al., p. 6 de ce numéro). Des squelettes d'individus périnataux, ou des dents de lait sont des indices plaidant en faveur d'un élevage in situ différent d'un simple abattage. Par crainte d'épizootie, peut-être par mesure d'hygiène ou parce qu'ils sont consommés, les animaux morts sont toutefois rarement retrouvés dans un bâtiment à destination d'élevage. Enfin, si des assemblages de restes végétaux permettent au carpologue de supposer un élevage sur site, les graines sont souvent rejetées à l'extérieur des bâtiments. Dans les rares cas où elles sont associées à un bâtiment, se pose alors la question de savoir si celui-ci était destiné au stockage du fourrage ou des bêtes... et de quelle espèce animale il s'agissait.

Toutes ces études «bonnes à penser » ${ }^{\mathbf{1}}$ nécessitent de conditions matérielles satisfaisantes de préservation et de fouille, rarement réunies. Elles ne dispensent de toute façon pas d'une étude archéologique poussée, intégrant un questionnement «agronomique» des vestiges. Pour reconnaitre une écurie ou une porcherie à l'état de vestiges, il faut commencer par savoir à quoi ressemblent de tels bâtiments lorsqu'ils sont encore en élévation dans nos régions. Nous avons en ce sens entrepris une double démarche depuis plus de dix ans en Île-de-France, en observant et relevant autant 
que possible les bâtiments (fermes et autres) avant démolition, et en les confrontant à de nombreux manuels anciens d'agriculture, traités nous décrivant les principes de fonctionnement et de construction des bâtiments agricoles. De cette littérature agronomique, relativement riche depuis le $\mathrm{XVI}^{\mathrm{e}}$ siècle, nous prônons une «lecture archéologique»; nous extrayons de ces lignes, pages ou chapitres, ce qui rejoint l'étude archéologique après abandon des bâtiments. Cette «théorie » de la construction agricole, fondée sur l'observation, nous permet de faire des propositions pour reconnaitre et interpréter le logement animal (entre autres) parmi nos vestiges archéologiques (Fechner et al., 2011). Associée aux études de faune, flore et sol, elle permet de préciser la fonction des bâtiments, et donc d'aller parfois plus loin dans l'économie rurale du site.

\section{Présentation des sources}

Sur 300 manuels anciens dépouillés, 65 ouvrages ${ }^{2}$ (et 3 articles) nous ont donné des informations sur le logement des principaux bétails. Nous ne prétendons certes pas avoir tout lu, mais sans doute les principaux titres. Hormis quatre auteurs antiques, ces ouvrages sont publiés entre le $\mathrm{XVI}^{\mathrm{e}}$ siècle et 1940. Ils nous donnent un bon aperçu de ce que furent les normes traditionnelles de construction pour le logement animal aux périodes moderne et contemporaine, et indirectement pour les périodes historiques antérieures. En effet, si l'on compare les prescriptions des quatre auteurs antiques à celles des modernes, on constate que les recommandations diffèrent très peu. Malgré l'écart temporel, les contraintes du logement animal restent les mêmes. Ces 66 références sont le fait de 63 auteurs. Nous avons pu suffisamment nous informer sur 54 de ces auteurs (86\%) pour soumettre leur texte à la critique. Si la grande majorité d'entre eux peuvent vraiment être qualifiés d'agronomes, huit ne sont toutefois que des compilateurs: Auguste Anex (1857), FrançoisAlexandre Aubert de la Chesnaye des Bois (1751), Jean-François Bastien (1798), Théodore Château (1863), l'abbé de Chanvallon (1780), l'imprimeur Giroud (1695), Varron et Benjamin Véret (1870).

Par la somme de leur travail, ou par sa diffusion, les textes de ces compilateurs sont toutefois utilisables car ils reflètent strictement ce qui se pratique ou s'écrit à leur époque. D'ailleurs, dans sa somme agronomique en 8 volumes in-quarto (plus 2 volumes posthumes), l'abbé Rozier, célèbre encyclopédiste, n'hésita pas à compiler les textes d'auteurs plus pointus que lui sur certains sujets. À l'opposé, certains agronomes sont aussi des expérimentateurs : Arthur Young (1772) ou Henri Louis Duhamel du Monceau (1779) décrivent parfois des techniques expérimentées sur leurs propres domaines, et qui ne sauraient refléter l'usage courant de l'époque. Il en va de même pour la description de certains bâtiments modèles, telle la porcherie d'engrais de Grignon, la bergerie nationale de Rambouillet...
Parmi ces 54 auteurs, un tiers a des compétences ou des fonctions liées à l'agriculture : ils sont membres d'académies agricoles, de sociétés d'agriculture...

Huit auteurs sont reconnus comme des agriculteurs praticiens : Edme-Jean Collot (1851), Columelle, Élysée Lefevre (1834a et b), Louis Moll (1834), l'abbé Rozier (1785 et 1805), Amand-Joseph SaintFélix de Maurémont (1826), Olivier de Serres (1600) et Arthur Young. Huit auteurs sont médecins, vétérinaires ou chimiste. C'est important, car les notions d'hygiène ou d'absence d'hygiène deviennent plus que récurrentes à propos des bâtiments d'élevage au cours des siècles. Dix auteurs sont architectes, spécialisés dans la construction rurale ou classique. Il est à noter que les architectes dits «classiques» utilisés ici - Charles-Étienne Briseux (1743), Augustin Charles Daviler (1710), Louis Savot (1625) et Vitruve - ont essentiellement un discours sur l'écurie, logement pour des animaux de choix. Le monde du cheval génère une littérature spécialisée qui traite de l'écurie. Trois auteurs, écuyers ou directeurs de haras, comptent parmi nos sources. Enfin, huit auteurs ont la qualification d'ingénieurs, six de professeurs des grandes écoles d'agriculture nationale, cinq ont une carrière politique importante, quatre sont religieux, trois militaires, un botaniste et un journaliste. Si tous ces hommes représentatifs de lélite de leur époque ont un discours agronomique, c'est bien parce que l'agriculture reste, dans toute l'Europe, la base de l'économie d'une grande majorité de la population contemporaine.

Les trois-quarts de ces ouvrages sont publiés à Paris, ce lieu d'édition étant pertinent comme secteur d'observation des auteurs. Parce que le «modèle» économique prédominant chez les agriculteurs aux époques moderne et contemporaine est celui de la grande culture céréalière, nous avons plus précisément eu recours à une littérature «françoise», c'est-à-dire francilienne. Cette origine d'édition donne une première limite à l'utilisation de ces manuels : celle de la grande culture étendue au bassin parisien, aux frontières certes larges, mais pas au-delà. Les caractères techniques et fonctionnels énoncés sont largement utilisables dans une bonne moitié nord de la France, mais certainement pas en milieu méditerranéen ou montagnard. Un quart des ouvrages consultés est édité dans d'autres grandes villes de l'Ouest européen (à Toulouse, à Lyon, en Belgique, à Leipzig). Ils nous assurent d'une couronne d'agronomes ayant travaillé et édité dans des terres différentes et nous apportent un recouvrement d'information plus large, et finalement des données très proches de celles issues des observations franciliennes.

Quelle est la diffusion réelle de ces auteurs? La grande majorité des manuels utilisés a connu un nombre d'éditions inférieur à cinq. Seuls le Théâtre d'agriculture (Serres, 1600) L'Agriculture et maison rustique (Estienne et Liebault, 1583) et la Maison rustique des dames, du seul auteur féminin de notre corpus, Cora Millet-Robinet (1893), ont 

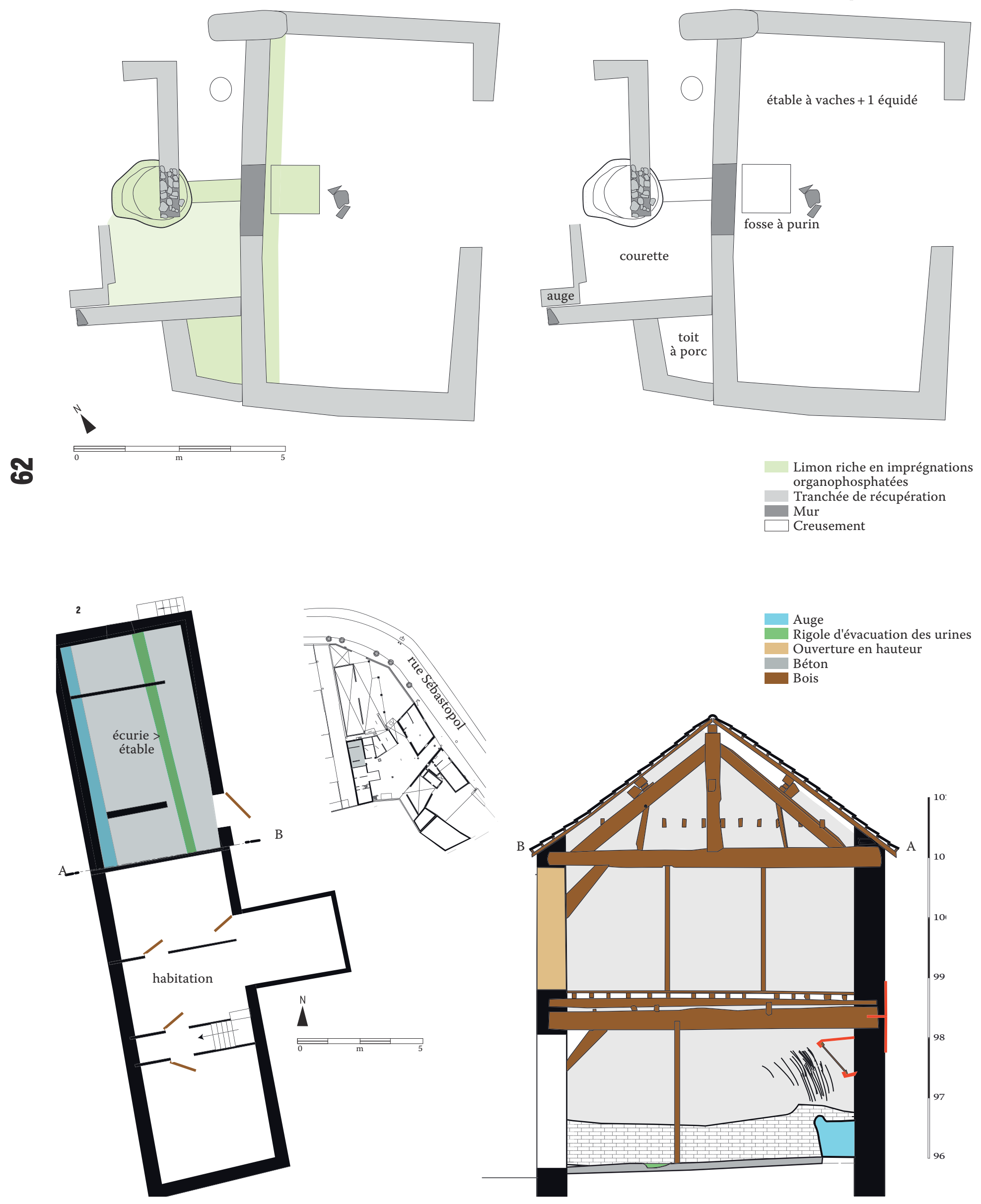
connu plus de 20 éditions. Ces chiffres sont bien faibles par rapport à la diffusion des auteurs antiques (250 éditions de Columelle), des écrits desquels tous nos auteurs modernes sont pétris. Il est difficile de savoir si, en dehors des élites des grands propriétaires terriens cultivés, ces traités eurent une réelle influence sur le monde paysan. Il est plus crédible d'expliquer que ces ouvrages sont essentiellement issus d'observations des techniques qui leur sont contemporaines, et rendent compte d'une certaine réalité paysanne. La plupart des ouvrages commencent d'ailleurs par décrire une situation jugée déplorable, des bâtiments d'élevage sales, sombres et humides... pour prescrire ou rappeler des normes de construction plus efficaces ou hygiéniques.

\section{Présentation synthétique des recommandations des manuels d'agronomie}

Des 66 sources agronomiques, nous avons extrait 31 «notices » sur la porcherie, 32 sur la bergerie, 34 sur l'écurie et 35 sur l'étable à bovins. Ces 132 mentions descriptives, lues avec un regard d'archéologue, nous permettent de rendre compte de programmes différents pour la construction de chaque type de logement animal. Signalons que 35 manuels, spécialisés ou non, ne sont utilisés que pour un seul type de logement ; 24 ouvrages sont utilisés dans trois ou quatre cas : les manuels «généraux» d'agriculture et les traités de construction rurale sont les principaux types de références avec les encyclopédies.

Par souci de clarté, nous présentons dans un premier temps chaque type de bâtiment. Pour une économie de leur construction, un confort du bétail et un accès pratique pour l'exploitant, tous les bâtiments d'élevage sont mesurés. Les données chiffrées sont issues de moyennes.

Les porcheries, ou «toits à porcs», présentent des sols dont l'aménagement retient une forte attention de la part des agronomes (23 occurrences). Ces sols doivent être en pente (14), ou surélevés (3) avec un caniveau pour l'écoulement des urines (7) et donc étanches (3), pavés (12), revêtus de briques (3), de ciment (2), en dalles (3) ou béton (2), ou encore planchéiés à claire-voie (6) avec du sable (2). Le second critère d'identification d'une porcherie réside dans la présence d'une cour associée (19 manuels) réduite à quelques mètres carrés qui permet au porc de se dégourdir et de se vider. Elle peut être pavée (5), agrémentée d'un bassin ou d'une mare (5), plantée d'arbres (3) et entourée de murs (2). Le fort compartimentage des animaux est une caractéristique majeure de la porcherie (17 auteurs). Les porcs doivent, en effet, être séparés selon leur âge, leur sexe, ou leur destination (engrais, reproduction). Ces compartiments du logement porcin sont, comme les séparations des courettes, construits en dur. Quinze auteurs précisent des dimensions différentes des cahutes ou toits à porcs, selon qu'il s'agit de porcs à l'engrais $\left(2,5 \mathrm{~m}^{2}\right)$, de verrats $\left(3,1 \mathrm{~m}^{2}\right)$, de truies pleines $\left(3,3 \mathrm{~m}^{2}\right)$ ou avec ses petits $\left(8,7 \mathrm{~m}^{2}\right)$. Les dimensions des bâtiments décrits ou fouillés sont donc pleinement significatives [ill. 1]. Dix auteurs traitent des ouvertures des toits pour les porcs. Les portes sont de faible largeur : $0,85 \mathrm{~m}$ en moyenne. Les auges des cochons sont bien décrites par 15 auteurs : placées à moitié dessous, à moitié en dehors du toit à porcs, de façon à pouvoir être remplies sans pénétrer dans la cahute (10 occurrences). Elles sont de plus encastrées dans les murs (5). Les toits à porcs sont donc de petits bâtiments très solides et cloisonnés, avec de nombreuses caractéristiques de construction susceptibles de laisser des traces au sol, et donc d'être archéologiquement repérables.

Une importance fondamentale est également apportée au sol des écuries ou étables à chevaux (28 mentions), qui doit être en pente avec une rigole (23 mentions), surélevé (12), pavé (22), planchéié (3) ou couvert de salpêtre (3), car un cheval au pied endommagé est inutilisable. Ces types de sols sont perceptibles par les archéologues [ill. 2]. Les dimensions des écuries sont très réfléchies. Une écurie simple mesure 5,1 $\mathrm{m}$ de largeur dans œuvre, une écurie double, $8,5 \mathrm{~m}$. Une largeur de 1,5 $\mathrm{m}$ par cheval étant prévue, cette donnée entre en compte pour fixer la longueur de l'écurie. Des séparations, boxes ou stalles, sont mentionnées dans 14 ouvrages. Les ouvertures d'écurie sont décrites par 22 auteurs. Les portes ont une largeur comprise entre 1,25 et 1,5 $\mathrm{m}$. Les ouvertures se font au nord (7) ou au midi (6). Enfin, 13 manuels abordent, avec des divergences, l'exposition des bâtiments. Dans tous les cas, il s'agit de protéger des vents. La seule difficulté dans l'identification des bâtiments d'élevage du grand bétail réside dans la maigre différence entre l'écurie et l'étable à bovins. Le terme commun d' «étable» englobe, à l'époque moderne, aussi bien le logis des bovidés que celui des équidés.

Les points les plus fréquemment exposés en ce qui concerne l'aménagement du logement des bovins ont trait au sol (3o mentions). Il est préférentiellement en pente (19), en dur (21), généralement pavé ou recouvert de sable, ou simplement constitué de terre battue (6). La pente favorise l'évacuation des urines. Les 16 mentions de ces rigoles apparaissent dans les textes à partir de la fin du XVIII ${ }^{\mathrm{e}}$ siècle. Le deuxième critère par ordre de citation (23) est celui des dimensions des étables, c'est-à-dire, pour les agronomes, leur largeur. Les manuels modernes recommandent de diviser l'espace dans la largeur entre un couloir d'accès côté mangeoire, la longueur de l'animal et un couloir arrière destiné au nettoyage. Une étable simple mesure en moyenne $4,5 \mathrm{~m}$ dans œuvre contre $8 \mathrm{~m}$ pour une étable double $(1,4 \mathrm{~m}$ de largeur par bovin). Cette moyenne masque naturellement des différences entre vaches laitières et bœufs à l'engrais, petite et grande race... L'exposition du bâtiment est le troisième critère retenu (18 mentions). Les agronomes préconisent une exposition au midi (9), ou au levant (10). En ce qui concerne les portes (14 mentions), la largeur 


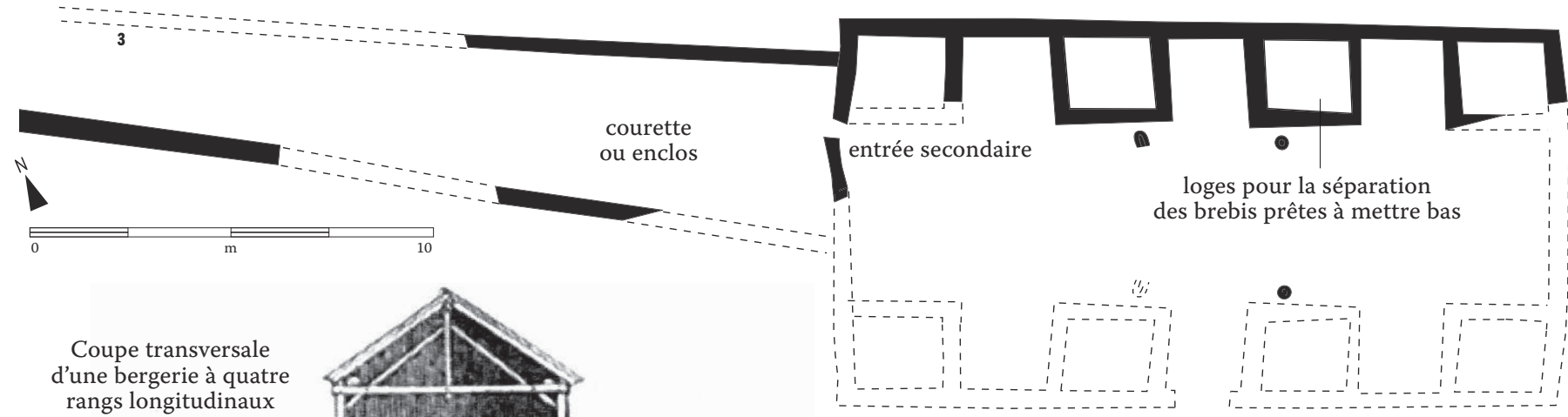
rangs longitudinaux

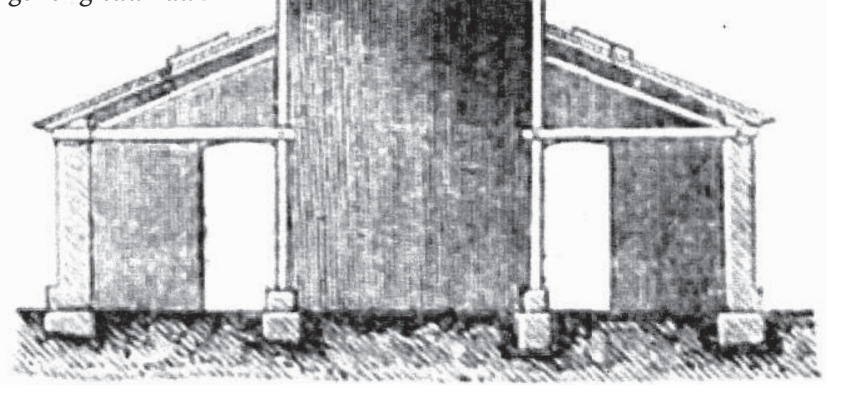

entrée principale
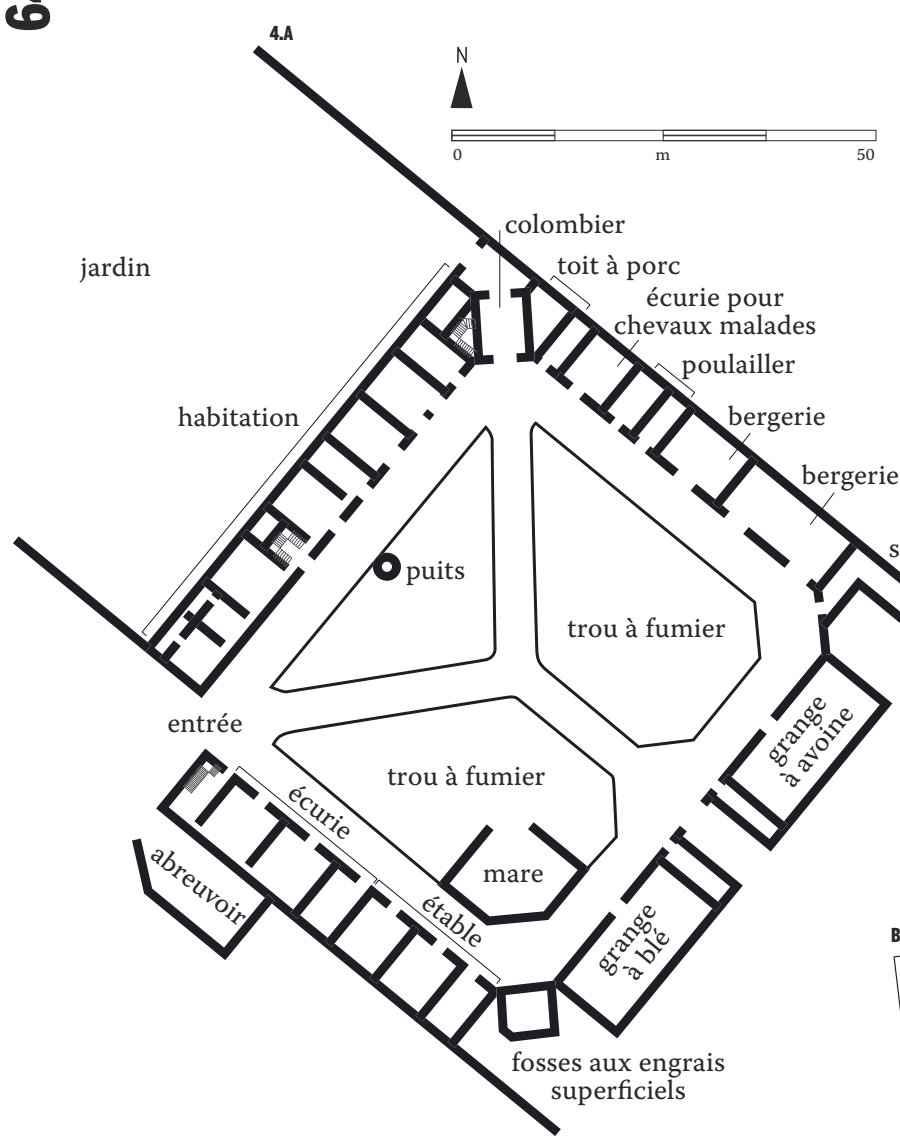

3. La bergerie, hors
standard, dans la bassecour du château de Roiss en-France (fin du xve s.), abritait 300 têtes d'ovins (26,6 x 14,5 m hors œuvre et $335 \mathrm{~m} 2$ ) Les (d'environ 3,15 x 2,6 m) permettent l'isolement ou la réunion d'animaux et facilitent la distribution de nourriture. Les phytolithes de graminées $(94,8 \%$ et $85 \%$ ) suggèrent un stockage céréalier (étude P.Verdin, Inrap, CEPAM).L'enrichissement en phosphore total a été important (étude C. Cammas, Inrap, AgroParisTech).
4. Comparaison entre le modèle de Perthuis (A) et le plan de la ferme Sassinot (B) à Roissy-enBrie (Seine-et-Marne). A. Écuries exposées nord-est, près de l'habitation, prolongées par les étables;

bergeries ouvertes au sud-ouest dans un angle de la cour ; toit à porc très réduit, au nord.

B. Écuries proches de la maison, prolongées par les étables ; bergeries pour moitié ouvertes

au nord ; porcherie ouverte et exposée au nord. Dans la seconde moitié du xIx's.,

le développement de la culture de la bettrave nécessitant des attelées de bovins et la diminution de l'élevage ovin en Île-de-France, une bergerie a été

réaménagée en étable à bovins.
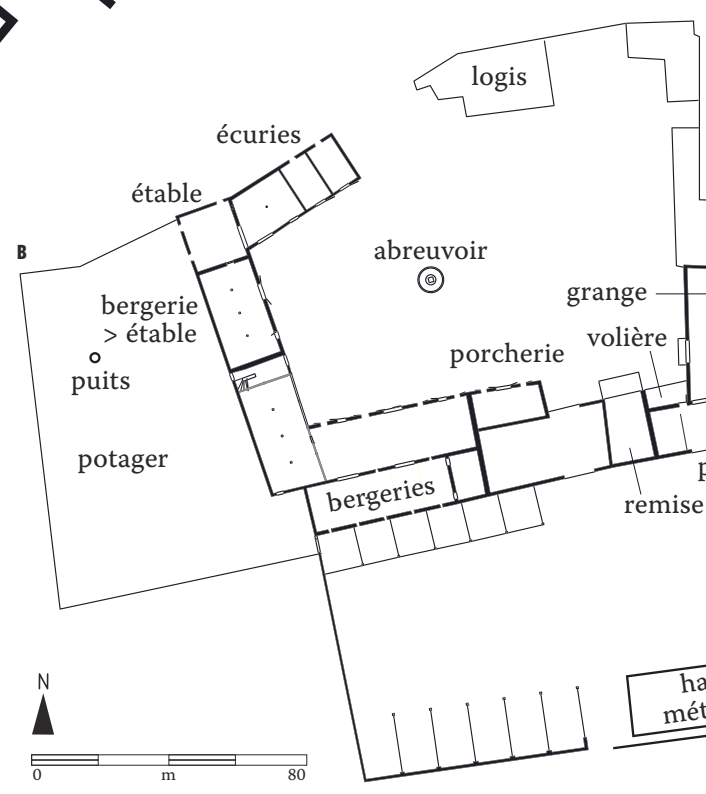

breuvoir

(a)

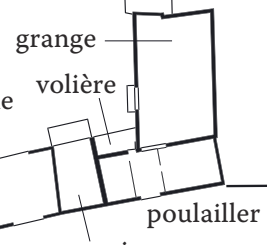

remise

vergers 
moyenne est de 1,6 m. Deux auteurs contemporains rappellent que les bœufs travaillant et se déplaçant par paire, les ouvertures doivent être prévues en conséquence. Pour l'équipement intérieur de l'étable, 8 auteurs sont favorables au compartimentage et 2 sont contre. Le lit du vacher (3 mentions) et l'âtre, évoqué par le seul Palladius, ne constituent pas des critères distinctifs suffisants.

La bergerie se distingue facilement [ill. 3]. Le premier critère est celui de l'exposition (21 mentions) : vers le midi (16) ou vers le levant (5). Columelle (VII, 3) nous explique le choix de cette exposition plein sud: «On les exposera au midi, parce que ce bétail, quoique le plus vêtu de tous les animaux, est cependant celui qui s'habitue le moins au froid, ainsi qu'aux chaleurs de l'été. » Le sujet de la «chaleur » des bergeries est très controversé et débattu parmi les agronomes, mais la majorité des auteurs s'en tiennent à cette exposition: "Les moutons ont besoin de soleil pendant l'hiver et d'ombre pendant l'été; la bergerie aura donc, si faire se peut, deux façades, l'une au midi et l'autre au nord. » (Cunisset-Carnot, 1890, p. 176) Le sol de la bergerie ( 21 mentions) est en terre battue (13), en pente et/ou surélevé (11), parqueté (2), en dur (8). Seuls cinq auteurs sont contre le pavement. «On ne pave point le sol des bergeries, et on ne lui procure aucun égout, parce que le fumier de mouton demande à être saturé d'urines » nous explique Perthuis de Laillevault (1810, p. 148). Les portes de la bergerie doivent être larges de 1,75 m, ouvertes au nord (3) ou au midi (3). Le cloisonnement intérieur de la bergerie (compartiments pour animaux de même âge, sexe ou régime...) est abordé par 15 manuels. Les dimensions (13 mentions) données sont de 4,4 $\mathrm{m}$ de largeur pour une bergerie simple, et de $7 \mathrm{~m}$ pour une bergerie double.

\section{Synthèse comparative}

De ces critères d'identification présentés pour les différents logements à bétail, il faut avant tout remarquer l'absence de toute longueur significative. Pour tous les auteurs, la longueur du bâtiment de stabulation dépend du nombre de tête que l'on doit y loger ; elle est donc extrêmement variable et paraît ainsi peu pertinente en tant que critère d'identification. Une écurie se construira avec les mêmes normes pour un cheval ou pour 10 chevaux. La longueur, souvent le premier critère abusivement utilisé dans l'analyse archéologique pour associer une fonction agricole à un bâtiment, ne paraît pas être le critère le plus pertinent pour cette identification. C'est la largeur du bâtiment qui compte. Cette donnée dépend partiellement de la longueur des animaux mis en stabulation. Celle-ci peut varier au cours des époques, tout comme leur hauteur au garrot. À ce jour, les méthodes employées par les archéozoologues ne s'intéressent pas à la restitution des longueurs des espèces domestiques. Les mesures données dans cet article ne sont qu'une moyenne, qui masque des disparités selon les races, et le type de bétail ; une génisse prend moins de place qu'une vache laitière, qui elle-même nécessite un espace moindre que celui du bœuf à l'engrais... Dans l'aménagement des loges à bétail, les auteurs anciens pallient les différences de hauteur de garrot en jouant sur la hauteur des mangeoires et râteliers.

Les sols des bâtiments d'élevage sont bien une préoccupation majeure des agronomes. On ne pouvait espérer situation plus favorable à l'analyse archéologique. Les bergeries se distinguent donc très facilement des autres logements à bestiaux par l'absence de sol en dur, et une construction plus légère que celle réservée aux autres bétails. Leurs portes sont les plus larges, car les moutons non attachés dans la bergerie tendent à se précipiter tous ensemble vers la sortie. À l'autre extrémité, les porcheries sont les bâtiments les plus compartimentés de la ferme et ceux qui requièrent la plus grande solidité. Leurs portes sont étroites. «Il faut donner beaucoup de solidité à tous les détails de construction d'un toit à porcs, car il n'y a pas d'animal plus destructeur que le cochon.» (De Fontenay, 1836)

L'écurie est globalement plus large que l'étable à bovins, et présente un sol plus fréquemment fait en dur, supportant plus souvent un cloisonnement Une étable est dotée de portes sensiblement plus larges, et répond à des critères d'exposition nets. Les mangeoires sont également plus basses et plus larges dans l'étable à bovins que dans l'étable à chevaux. Nous touchons ici une autre limite des données exposées dans cet article. Nous avons volontairement rendu compte d'informations facilement perceptibles en plan. Une véritable archéologie du bâti agricole, telle que nous tentons de la mettre en œuvre, fait intervenir d'autres critères liés à l'élévation du bâtiment : forme et position des fenêtres, nature du couvrement...

\section{Aménagement de l'habitation rurale}

Si chaque bétail nécessite un bâtiment d'élevage avec des normes intrinsèques, la vie des hommes avec les bêtes suscite une réflexion plus générale concernant l'aménagement de la ferme ou de la métairie [ill. 4].

Dans l'introduction de son essai de classification, Albert Demangeon précise que la véritable originalité de l'habitation rurale en France réside non pas dans ses matériaux ni dans ses formes extérieures, mais dans son plan, dans la répartition établie entre les animaux, les hommes et les choses (Demangeon, 1920, p. 353-354). Divers agronomes anciens proposent des modèles de distribution des bâtiments au sein d'une ferme en pays céréalier. Ces modèles globaux de distribution sont destinés à augmenter le côté pratique, la productivité et la rentabilité du travail des hommes et des animaux au sein d'une agriculture raisonnée. Parce qu'elle obéit à des règles plutôt codifiées, la distribution des bâtiments d'élevage au sein d'une ferme participe 
également à leur interprétation. La plupart des modèles de distribution lus positionnent l'écurie à proximité immédiate du logement du propriétaire ou de l'exploitant. Donc, si l'étude archéologique identifie facilement la maison des hommes, celle du cheval n'est pas loin. Les bovins sont souvent logés dans la continuité des chevaux, en témoigne, parmi d'autres, l'abbé Louis Rose : «La disposition la plus avantageuse d'une étable des vaches ou vacherie, est toujours celle où elle se trouvera le plus près possible de la cuisine; E cela pour la commodité des porteurs de breuvage $\mathcal{E}$ laitages; E lorsqu'elle sera attenante à l'écurie pour la facilité du mélange des fumiers. » (Louis Rose, 1767, p. 101) Depuis le XVI ${ }^{\mathrm{e}}$ siècle, les porcheries ne sont pas importantes en pays de grande culture. Elles sont ouvertes au nord dans le modèle de Charles Estienne (1564), réduites à un toit à porc disposé dans l'angle nord dans le modèle de Thouin au début XIX $^{\mathrm{e}}$ siècle, et mises sous le vent de la cour en 1898 par Maximilien Ringelmann (1893). Enfin, la présence d'aménagements extérieurs significatifs peut représenter un critère déterminant pour identifier un bâtiment de stabulation, voire pour reconnaître l'espèce parquée. Parmi les structures les plus souvent évoquées dans les manuels, se retrouvent la laiterie, la pièce pour la préparation des aliments du bétail et surtout la fosse à fumier. Celle-ci, contrairement à d'autres infrastructures liées à l'élevage (mare, bassin de lavage, abreuvoir, etc.), est nécessairement localisée à proximité immédiate du logement des animaux. Rendues inutiles par le développement des moyens mécaniques d'évacuation, les fosses à fumier sont souvent absentes de nos observations archéologiques avant démolition. Elles sont malgré tout très facilement repérables archéologiquement.

Reconnaître le logement animal s'avère possible, notamment dans les régions où le modèle de la maison sur cour domine. Ce modèle d'habitat paysan groupe les bâtiments spécialisés par fonction autour d'une cour, et les rend donc plus lisibles pour l'archéologue. Ce modèle est celui de la villa antique, aussi les critères de distinction énoncés nous semblent-ils largement applicables pour cette période. Ainsi à Saint-Brice-sous-Forêt (Val-d'Oise), les analyses au sol et les observations agronomiques ont permis l'identification d'une étable à bovins sur le site antique de La Chapelle Saint-Nicolas (Rouppert et al., 2011). La connaissance préalable nécessaire à la reconnaissance du logement animal montre toutefois des variantes régionales. Les maisons en hauteur des provinces méditerranéennes, ou les maisons-blocs de la France de l'Est obéissent à d'autres règles de distribution qui ne sont pas abordées dans cet article. Les bâtiments mixtes ont toutefois pu exister à différentes époques, parallèlement aux dispositions étalées. La maison de Roissy-en-France (Val-d'Oise) au XII ${ }^{\mathrm{e}}$ siècle [cf. encadré p. 67] en est un exemple. L'étable à pourceaux, qui flanque cette maison, ne répond pas à l'idée principale d'une porcherie telle qu'exprimée par les manuels, mais bien à une variante, appuyée sur des critères peu fréquents, de faible occurrence dans les manuels d'agriculture. Ces traités écrits entre le $\mathrm{XVI}^{\mathrm{e}}$ et le $\mathrm{XX}^{\mathrm{e}}$ siècles peuvent ainsi également rendre compte de situations bien plus anciennes.

\section{Références bibliographiques}

Columella, 2001 (réédition de 1941), On agriculture, Books I-IV, translated by H. Boyd Ash, Loeb Classical Library, Edimburg, St Edmundsbury Press Ltd, $441 \mathrm{p}$.

Digois E., 1942, Le Livre du bon moutonnier, Paris, La Maison rustique, ze édition, $406 \mathrm{p}$.

Palladius, 2003 (réédition de 1976), Traité d'agriculture, t. I (livres I-II), texte établi, traduit et commenté par R. Martin, Paris, Les Belles Lettres, 209 p.

VARRON, 2001 (réédition de 1978), Économie rurale. Livre premier, texte établi, traduit et commenté par J. Heurgon, Paris, Les Belles Lettres, 350 p.

VARRON, 2003 (réédition de 1985), Économie rurale. Livre II, texte établi, traduit et commenté par C. Guiraud, Paris, Les Belles Lettres, $171 \mathrm{p}$

Vitruve, 1995 (réédition de 1684), De l'architecture, texte traduit par Claude Perrault, Paris, Bibliothèque de l'image, $329 \mathrm{p}$.

ANEX A., 1857, La laiterie du village et le train de la fromagerie, Lausanne, S. Blanc libraire-éditeur, 208 p.

Aubert de la Chesnaye des Bois F.-A., 1751, Dictionnaire universel d'agriculture et de jardinage, defauconnerie, chasse, pêche, cuisine et manège, 2 vol., Paris, David, 730 p. et $467 \mathrm{p}$.

BAstien J.-F., 1798, La Nouvelle Maison rustique ou Économie rurale pratique et générale de tous les biens de campagne, 3 vol., Paris, 889, 899 et 1006 p.

Briseux C. E. 1743, L'Art de bâtir des maisons de campagne, 2 vol., Paris, 257 p. et $260 \mathrm{pl}$.

Château Th., 1863, Technologie du bâtiment, I, Paris, Librairie d'architecture de B. Bance, $517 \mathrm{p}$

Collot E., 1851, Traitéspécial de la vache laitière et de lélève du bétail, Paris, $500 \mathrm{p}$.

CUnisset-CARNOT P., 189o, Le Livre d'agriculture. Lectures agricoles. Excursions. Expériences. Rédaction, problèmes et dictées sur l'agriculture Paris, Librairie Larousse, 4 édition, 296 p.
Daviler A. C., 1710, Cours d'architecture, 3 vol., Paris, $920 \mathrm{p}$.

De Chanvalon abBÉ, 178o, Manuel des champs, ou recueil amusant et instructif concernant tout ce qui est le plus nécessaire E le plus utile, pour vivre à la campagne avec aisance E agrément, Paris, Lottin le jeune, 578 p.

De FontenAy M., 1836, Manuel pratique des constructions rustiques, Paris, librairie encyclopédique de Roret, 594 et $779 \mathrm{p}$.

Duhamel du Monceau H. L., 1779, Éléments d'agriculture, 2 vol., Paris, 532 et 462 p.

Estienne C., Liebault J., 1698 (réédition de 1583), L'agriculture et maison rustique, Lyon, $362 \mathrm{p}$.

GIROUD A., 1695, L'Agriculture et ménagerie des champs et de la ville. Grenoble, $252 \mathrm{p}$.

Lefevre E., 1834a, « Du porc », in Bailly C., Bixio A. MALPEYRE F. (DIR.), Maison rustique du XIX $X^{e}$ siècle, t. II, Paris, Librairie agricole de la maison rustique, p. 488-497

LefeVre E. ,1834b, « Du mouton », in Bailly C., Bixio A., Malpeyre F. (DIR.), Maison rustique du XIX $X^{e}$ siècle, t. II Paris, Librairie agricole de la maison rustique, p. 501-511.

Millet-Robinet C., 1893, Maison rustique des dames, Paris, Librairie agricole de la maison rustique, $14^{\mathrm{e}}$ édition, 681 et $664 \mathrm{p}$

MolL L., 1834, « De la nourriture des bêtes bovines », in Bailly C., Bixio A., Malpeyre F (Dir.), Maison rustique du XIX $X^{e}$ siècle, t. II, Paris, Librairie agricole de la maison rustique, p. 468-481.

Perthuis de LAIllevault L. de, 1810, Traité d'architecture rurale, Paris, 263 p., 25 pl.

Ringelmann M., 1893, De la construction des bâtiments ruraux. Les bâtiments de la ferme, Paris, librairie Hachette et Cie, $271 \mathrm{p}$.

Rose L., 1767, La Bonne Fermière, ou éléments économiques utiles aux jeunes personnes destinées à cet état. Lille, imprimerie de J.B. Henry, 205 p.
ROZIER ABBÉ, 1785, Cours complet d'agriculture, t. VI, Paris, rue et hôtel Serpente, $735 \mathrm{p}$.

Rozier Abbé, Chaptal J.A.C., Parmentier A.A., Delalanse, Mongez, Lasteyrie, Dussieux, Gilbert, Rougier de la Bergerie, Thouin A., 1805, Cours complet d'agriculture, t XI, Paris, $492 \mathrm{p}$.

SAvot L., 1685 (réédition de 1625), L'Architecture française des bâtiments particuliers, Paris, $436 \mathrm{p}$.

SERrEs O. DE, 1996 (réédition de 1600), Le Théâtre d'agriculture et mesnage des champs, Paris, Actes Sud, $1410 \mathrm{p}$.

Saint-Félix Mauremont A.-J.-M., 1826, Architecture rurale, théorie et pratique à l'usage des propriétaires et des ouvriers de la campagne, Toulouse, $395 \mathrm{p}$.

Thä̈R A. D., 1831, Principes raisonnés d'agriculture, vol. IV, Genève, Paris, A.B. Cherbuliez, 724 p.

VÉRET B., 1870, Le Livre du village. Agriculture, jardinage, économie rurale, Amiens, Lambert-Caron imprimeur-libraire, $363 \mathrm{p}$.

Young A., 1772, Le Guide du fermier ou instructions pour élever, nourrir, acheter $\mathcal{E}$ vendre les bêtes à cornes, les brebis, les moutons, les agneaux E les cochons, Paris, J.P. Costard, 259 et 258 p.

DEMANGEON A. 1920, « L'habitation rurale en France. Essai de classification des principaux types », Annales de géographie, vol. XXIX, n 161, p. 352-375.

ROUPPERT V., Dufour J.-Y., FeCHNER K., 2011, " An example of a fruitful discussion between a pedologist and an archaeologist. A $1^{\text {st }}-4^{\text {th }}$ Century $\mathrm{AD}$ agricultural enclosure with a stable and a manure pit at "Chapelle Saint-Nicolas" in SaintBrice-sous-Forêt (Val-d'Oise, France) », in Fechner K., Devos Y., Leopold M, VölKel J. (ÉDS.), Archaeology, Soil- and Life-Sciences applied to Enclosures and Fields, BAR International Series 2222, p. 133-142. 